\title{
Second Screen Comes to the Silver Screen
}

\author{
A Consumer Study Regarding Mobile Technologies in the Cinema
}

\author{
Jonathan Weinel \& Stuart Cunningham \\ Creative and Applied Research for the Digital Society (CARDS) \\ Glyndŵr University, UK \\ \{j.weinel | s.cunningham\}@glyndwr.ac.uk
}

\begin{abstract}
Second screen' technology is the use of mobile technologies and other computing devices in order to provide an additional screen that supports or augments the consumption of visually based media. The 'Second Screen comes to the Silver Screen' project sought to explore the feasibility of using second screen context within a real-time cinema context, in order to augment and enhance the audience experience. The project included a review of technological feasibility, a consumer survey and two case studies. The results of the consumer survey and case studies are presented in this paper, the conclusions of which indicate a significant level of audience concern regarding the potential distraction that would be caused by second screen technology within the cinema. Conversely, the study does indicate some appetite for the use of mobile technologies to improve the cinema experience more generally, indicating a possible basis for further investigation regarding how this can be achieved appropriately.
\end{abstract}

Keywords-Context-aware computing; Affective computing; Second screen; Internet of Things; Cinema

\section{INTRODUCTION}

'Second screening' is a general term that is used to describe the use of mobile technologies to provide supplementary media content in support of that received from a primary screen [1]. A typical example which occurs in many households, is the use of a smartphone in order to browse internet content, send text messages or carried other activities, while simultaneously using a larger TV screen [2]. In such cases, the activities being carried out using the 'second screen' (the mobile phone) may be either related or unrelated to what is happening simultaneously on the primary screen.

In recent years developers and advertisers have become particularly eager to exploit the benefit of second screening in order to capture audience interest, regardless of which media device they are using [3]. While a large amount of second screening activity takes place ad-hoc using the normal services provided by smartphones (e.g. web browsing, social media, or SMS), the commercial interests at stake have also led to a variety of applications which are designed specifically for the second screen experience. These include a variety of companion apps which are designed to accompany particular TV shows or films, and application such as Zeebox offer content that is synchronised with TV programming [4]. These applications can be seen as part of a broader emerging culture that delivers media content through multiple simultaneous screens, as also evidenced by games consoles such as Nintendo's 3DS and Wii U [5]. While still in its infancy, this second screen culture clearly holds many interesting possibilities not only for advertising and commercial enterprise, but also for novel and creative delivery of content.

The 'Second Screen Comes to the Silver Screen' project seeks to explore the potential for invigorating a lagging cinema industry using second screen technology. In recent years cinema attendance has suffered from the availability of both illegal downloading and legal streaming media services such as NetFlix and LoveFilm; these have drawn customers away from attending the cinema by increasing the convenience with which a wide range of content can be consumed from the comfort of ones own home [6]. In order to address this it was proposed that second screening technology could be investigated as a possible means to enrich, augment or enhance the cinema experience in a way which way tailored to the individual. This could help to provide a unique experience for cinema customers, extend the interest in the cinema experience and enhance the value of attending a cinema performance of a film.

In consideration of the above, Stuart Cunningham proposed the design shown in Fig. 1 and Fig. 2. Fig. 1 shows the theoretical model for the design, while Fig. 2 indicates a conceptual mockup. The technology was proposed to function by synchronising a mobile device with what was being shown at the cinema. This would then enable the application to deliver tailored content in support of the movie performance. Critically, this would include services for use both before and after a film, as well as real-time services that could be used during the feature presentation. Some proposed possible uses could include additional information regarding particular scenes in a film, as well as more functional features such as the facility to order drinks or popcorn. Considering 
the former, we could conceive of an action film in which a bomb timer within the film is displayed on a second screen, supporting the experience. Imagine watching Speed, but being able to see the speedometer at all times on an additional screen for example. Alternatively, films could provide trivia or other audience-poll related information; there are many possibilities.

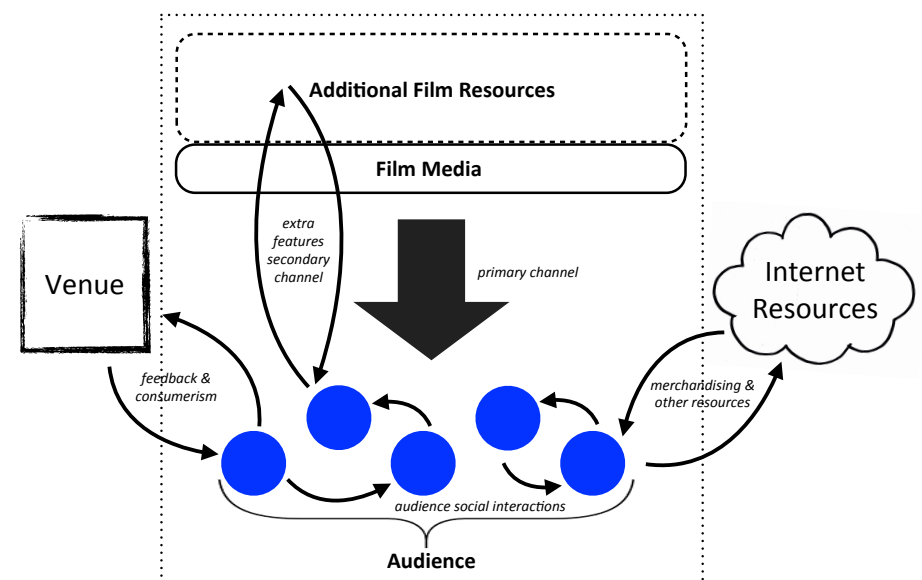

Fig. 1. Theoretical model in cinema context.

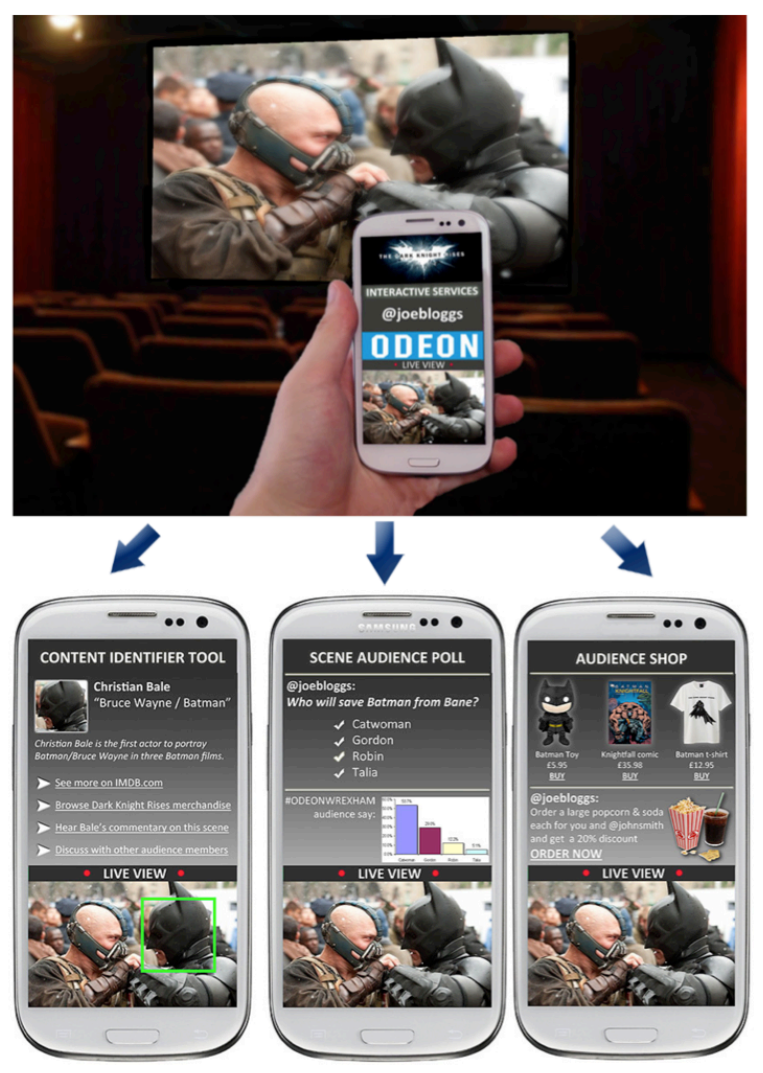

Fig. 2. Mock/conceptual model (Cunningham, 2013).

The purpose of the study was to investigate the feasibility of such a technology in the cinema context. As part of this study, a consumer study and two case studies were carried out at local independent cinemas.
The purpose of these was to explore the feasibility of the technology from the perspective of both cinema customers and cinema workers. It was anticipated that the outcomes of this research would indicate whether the proposed design would be seen positively, and give some indication regarding how to approach any possible use of second screening within the specific context of the cinema.

\section{Methodology}

A consumer survey was carried out using Glyndwr students. This consisted of both a focus group held with 23 students, and an online survey that received 89 respondents. The focus group consisted of a presentation regarding the proposed technology, and an open discussion regarding the students' opinions and ideas relating to this. This produced qualitative data that was analysed to elicit the range of opinion, any recurrent themes, and reasons given for any particular views that emerged.

The online survey consisted of a questionnaire that was designed to investigate whether the participants would be keen to use a second screen technology within the cinema context and what features they might like it have. The opinions of a student population were perceived as potentially valuable for the study, as teenagers and young adults were predicted to be the group would be the most likely to be interested in using the proposed technology (this age group are the most likely to own mobile phones and go to the cinema).

A market survey was also devised, which was aimed at acquiring information regarding the proposed design from companies such as RealD, Odeon, Vue, Cineworld and IMAX, however all declined to participate. Therefore information regarding the market is limited to that provided via the case studies.

Two case studies were carried out at the Scala cinema in Prestatyn (North Wales) and at Stoke Film Theatre (Staffordshire). The case studies involved interviews with customers of the cinemas, owners and employees. The technical facilities of these cinemas were also surveyed.

Combined anaylsis of the qualitative data from the focus group and case studies, with respect to the quantitative data provided by the online survey was anticipated to give a sufficient indication of public opinion regarding the proposed technology.

\section{CONSUMER SURVEY}

\section{A. Focus Group}

The focus group revealed aspects of the current cinema that the students found dissatisfying. These 
included: noise from other punters and the expense of tickets. Product advertisements were seen as annoying, though movie trailers were considered enjoyable. In general the cinema was seen as social activity, but that often home-viewing was considered preferable due to the enhanced choice offered by streaming services.

The proposed second screen technology was seen as potentially distracting, although it was mentioned that for a second viewing of the same movie it might add additional interest. Social features; personalization of the cinema experience; reducing the annoyance of adverts; convenience of ordering refreshments; and improving accessibility for those with disabilities were features that were identified as potentially beneficial.

\section{B. Online Survey}

The online survey consisted of adult students across all ages and both sexes, but most predominantly within their late teens and twenties. Most owned a smartphone and visited the cinema at least once every 6 months, which they used for social networking, playing games and using other apps. Most respondents agreed that they used their phone to 'second screen' while watching TV, with email, texting, Facebook and instant messaging being the most popular activities.

More than half of the respondents stated that they would be interested in trying a second screen app for the cinema (Fig. 3). The following were among the most popular of the proposed/possible features: accessing information; extra content; exclusive discounts; and ordering refreshments (in order of preference) (Fig. 4). Critically however, the majority of respondents indicated that they would use such an app before the movie or after the movie; only $12 \%$ said that they would use such an app during the movie (Fig. 5). Similarly, 58\% indicated that they would dislike other members of the audience using a second screen app during the film (Fig. 6). Only $18 \%$ said that they would actively seek to book tickets to a film where second screening was available, with the rest indicating they would either actively avoid screenings where second screening was available, or that they didn't care either way.

\section{Would you be interested in trying a second screen app for cinema}

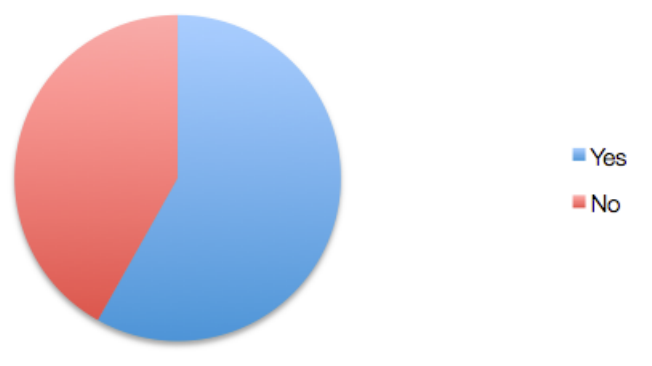

Fig. 3. Interest in trying a second screen app for the cinema.

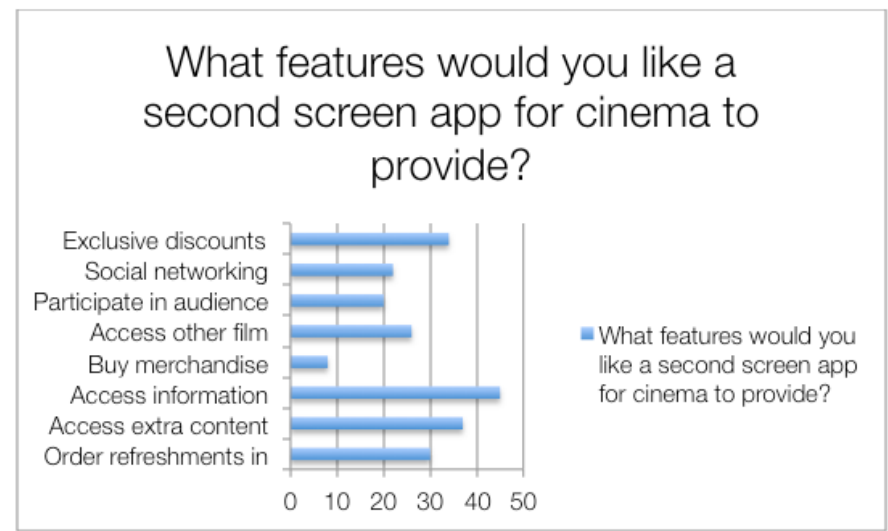

Fig. 4. Preferred features to see in a second screen app for the cinema.

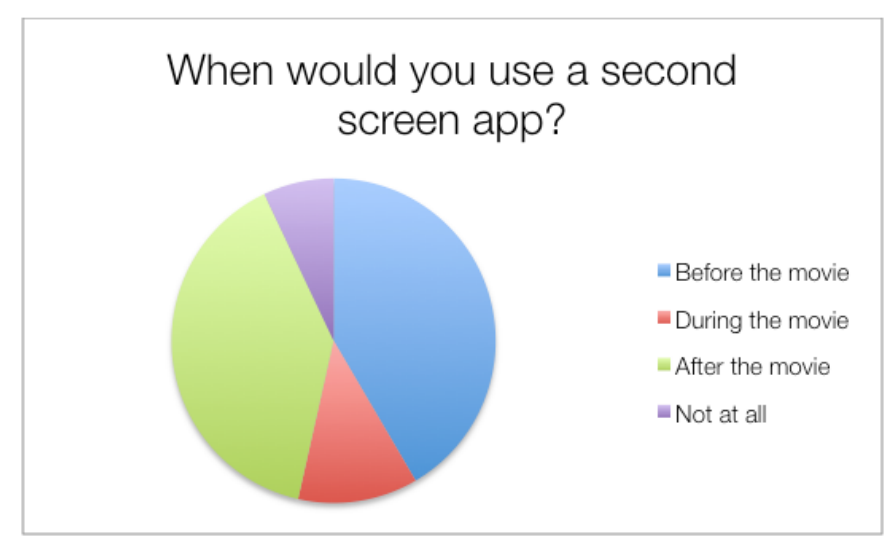

Fig. 5. When consumers say they would use a second screen app. 


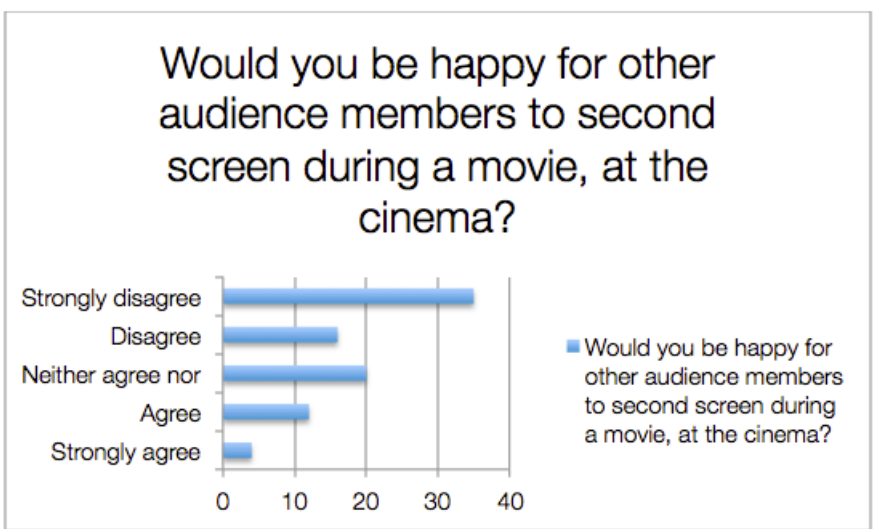

Fig. 6. Consumer expectation of comfort while others second screen.

In general, the negative response towards second screening was qualified by the free-text comments provided in the survey; many participants indicated that they would find second screening annoying or distracting during the actual film, though during trailers or in the lobby it would be acceptable.

\section{CASE STUdiES}

\section{A. Stoke Film Theatre}

Stoke Film Theatre is a small independent cinema which is part of the Staffordshire University campus in Stoke-on-Trent. It has one screen and presents a regular programme of mainstream, art-house and international films.

Our interview with the theatre's head of house prompted a very negative response to the proposed technology. She indicated that the customers of the theater would dislike the technology and see it as a distraction if it were used during the film. The film theatre had worked particularly hard to minimize distractions such as lighting in the theatre, and prohibits the audience from leaving until the credits are over; thus the proposed technology would be seen as particularly antithetical to the experience the cinema sought to offer:

"...a lot of people like to come to the film theatre because they can see the film with no interruptions... generally it's a place to see a film and actually watch the film, with people who also appreciate film... we certainly would feel, I think, that anything that was a distraction [to] the film, or anything that would disturb people viewing the film and destroy their enjoyment of the film, that it wouldn't be a good idea."

She did however indicate that a supporting technology tailored to the venue could have some benefits.
The customers of the cinema provided a similarly negative impression of the proposed technology. Most people saw it as potentially distracting for audience members to use a screen during the film. Older participants in the study responded in particularly negative terms, and while some younger members indicated they would be interested to try it, the predominant view was that it would be distracting and annoying:

"in quite dark cinemas its really noticeable if someone on your row or in front of you turns on their phone. When people check their phones and that brightness... you catch it out the corner of your eye and it is really annoying, you're like:- "turn it off!" But maybe before and after I'd use it more than actually in the cinema."

\section{B. Scala Cinema}

The Scala is an independent cinema and arts centre in Prestatyn, North Wales. It has multiple screens and presents a variety of major releases, as well as other forms of theatre and events.

The interviews we carried out with staff members at the Scala reiterated the concerns about distraction from the light of a mobile phone screen during the film, and the technology was seen as a fairly negative proposition. Another concern was also identified, which was the potential problems in detecting whether customers were using the approved technology, or whether they were recording video (which is prohibited due to copyright reasons):

"I see a couple of problems. The first one is that something film companies are always trying to do, and that we always do is check that nobody is trying to film with a camera or a phone.... [the] second one is when you see someone texting, people get annoyed, because of the light from the screen."

Interviews with the customers at the Scala (who were predominantly women in an older age range) indicated a generally positive attitude toward the idea of some form of new technology if it were used before or after the film, but echoed the sentiment that runs throughout this study that if used during a film, it would be perceived as a negative distraction: 
"I think [they should have it] before and after the film, I wouldn't like it during the film"

"I think it would be a good thing at the end of the film."

\section{CONCLUSION}

In conclusion, the report on technological feasibility that accompanied this study (discussed elsewhere in a forthcoming paper) indicated that the proposed second screening technology could be achieved technically, with synchronous content being provided using WiFi, RFID chips or audio watermarking. However, our consumer survey and case studies indicated a mainly negative attitude towards the concept of a second screening technology being used during films at the cinema. The main concern that most participants had was that the technology would be seen as distracting and annoying.

Despite this, our study did indicate that a mobile app for the cinema that was designed for use before and after the film could be appealing for users. As part of the study, several initial alternative design concepts were created which propose other types of context-aware interaction aimed specifically at cinema audiences. For example, an app could identify when the user is at the cinema, and use the knowledge of what they have seen or plan to see to provide specifically tailored content that enriches their experience and extends their interest in engagement. It would also be possible to consider methods through which an app for use outside of the cinema experience could involve collaboration with other local businesses that potentially benefit from a night out at the cinema, such as local restaurants (similar to the model used by Foursquare). As second screening during the film is likely to be distracting, we propose that future research focuses on these types of contextaware applications that focus on engaging audiences outside of the actual film experience.

\section{ACKNOWLEDGMENT}

This study was carried out with the support of the Glyndŵr University students who participated in the study, the staff and customers of Stoke Film Theatre and the Scala cinema. Since carrying out the research, the Scala cinema has regrettably closed its doors to the public; we wish them all the best for the future.

\section{REFERENCES}

[1] G. Winslow, "Broadcasters Sync Up Second Screen Efforts". Broadcasting \& Cable, 19 November 2012, pp.20-21.

[2] Deloitte, "TV: Why? Perspectives on TV: dual screen, catch up, advertising, and why people watch TV", 2012.

[3] PwC, "Entertaiment \& media outlook: The outlook for the Entertainment and Media industry to 2016", 2012.

[4] R. Andrews, "NBCU, Comcast buy in to Zeebox to take 'second screen' to prime time", paidContent, 2013.

[5] W. Greenwald, "Hands On: Nintendo's TVii on the Wii U", PC Mag, 2013.

[6] T. Kuittinen, "Netflix vs. movie theatres: Consumers staying at home more", BGR, $2^{\text {nd }}$ January 2015. 